\title{
An Adaptive Histogram Values \& Texture Descriptor for Data Analysis
}

\author{
Ritika Verma, Vinay Thakur \\ ${ }^{1}$ Sri Sai University, M.Tech CSE, Palampur, India \\ ${ }^{2}$ Sri Sai University, Astt-Professor ECE, Palampur, India
}

\begin{abstract}
Image Retrieval Systems is a useful method for pattern recognition and artificial intelligence. There are three methods used for image retrieval: such as Content based image retrieval, Text based image retrieval system and Semantic based image retrieval. In content based image retrieval system, images are stored by visual content, such as Texture, Shape, Layout and Color. Color, Texture, Shape and Layout are the primitive methods which are used in content based image retrieval system. In this paper presents a methodology which compares the images with the database using different types of parameters and gives the resulted image which is closely matched with the query image. For being this purpose using a five parameters for analysis image such as Entropy, Correlation, Standard Deviation, Contrast and Local Range. Histogram Values are used to extract the color properties of an image. The Combine feature of color and texture of images providing a robust feature for image retrieval. It achieves a experimental results which give the elapsed time for searching the image.
\end{abstract}

Keywords: CBIR, Texture Extraction, Color Histogram, Image Texture, Pattern Recognition

\section{Introduction}

An image retrieval system is those system which is used for browsing, searching and retrieving images from large database. In traditional some common methods are used to retrieve the images such as add metadata, caption or descriptor of images. This traditional method are time consuming and expensive. The trouble is took place in the main while verbal exchange between an records, photo seeker, person and picture retrieval device. Since the consumer may additionally have differing needs and understanding approximately the photograph series, an picture retrieval device ought to assist diverse forms for question formula. An image is basically an extended string of pixels for which every pixel is identified with the aid of its area within the photo matrix, its shade and its intensity. An analysis of the pixel set can give facts approximately the distribution of dominant colorations, the image texture, and the shapes formed by using marked change in neighboring shades In standard, photo retrieval queries can be labeled as:

- Attribute-primarily based queries, which use context and/ structural metadata values to retrieve photographs, for instance.

- Textual queries, which use a time period-based specification of the preferred images that may be matched to textual photograph descriptors.

- Visible queries, which give visible traits (shade, texture) or a picture instance that, can be compared to visible descriptors.

With the steady boom of laptop power, hastily declining value of storage and ever-growing get entry to to the internet, digital acquisition of statistics has turn out to be increasingly popular in recent years. Digital records is optimum to analogy codec's because of handy sharing and distribution houses. This fashion has inspired research in photograph databases, which have been nearly disregarded with the aid of conventional laptop structures due to the big quantity of facts vital to represent photos and the difficulty of mechanically reading photographs. presently, storage is much less of an issue considering the fact that big garage potential is available at low value. but, effective indexing and looking of big scale photo databases stays as a assignment for laptop structures. The content material based photo Retrieval system (CBIR) is a gadget, which retrieves the snap shots from an photo collection wherein the retrieval is based on a query, which is precise through content material and no longer by index or address. The query picture is an image wherein a person is interested and wants to find similar pix from the photograph collection.

The content material based image Retrieval device (CBIR) is a machine, which retrieves the pix from an photograph collection wherein the retrieval is primarily based on a query, that's specific with the aid of content and no longer by means of index or address. The question picture is an picture wherein a user is fascinated and wants to discover comparable images from the image series. The CBIR system retrieves relevant pics from an photograph collection primarily based on computerized derived functions.

The derived functions encompass primitive features like texture, shade, and shape. The features may also be logical features like identity of objects shown, summary features like importance of some scene-depicted and many others. there are many standard-cause image search engines. within the industrial area, IBM QBIC is one of the earliest evolved systems. recently, additional systems were developed at IBM T.J. Watson, VIRAGE, NEC AMORE, Bell Laboratory, Interpix (Yahoo), Excalibur, and Scour.net. in the educational domain, MIT Photobook is one of the earliest. Berkeley Blobworld, Columbia VisualSEEK and internet are trying to find, CMU Informedia, u.s.a. NeTra, united states of america, WBIIS are a number of the current systems. The proposed CBIR device can be extended at the alternative primitive characteristic vectors like, coloration and form [1]. 


\section{International Journal of Science and Research (IJSR) \\ ISSN (Online): 2319-7064}

Index Copernicus Value (2013): 6.14 | Impact Factor (2015): 6.391

Color is a feature of the first-rate majority of content material primarily based photo retrieval machine. but the robustness, effectiveness, and efficiency of its use in photo indexing are nevertheless open troubles. In photograph preprocessing, the capabilities used to represent coloration records and the measures adopted to compute similarity among the features of two pics are severely analyzed .characteristic extraction is very essential step in photo retrieval machine to explain the photograph with minimum wide variety of descriptors. The basic visual features of pictures consist of color and texture [3].studies in content material primarily based picture retrieval today is a active disciplined, increasing in breadth [4].representative functions extracted from images are stored in function database and used for object-based totally image retrieval. This document is a template. An electronic copy can be downloaded from the conference website. For questions on paper guidelines, please contact the conference publications committee as indicated on the conference website. Information about final paper submission is available from the conference website.

\subsection{CBIR (Content based image retrieval system)}

The sector of representing, organizing and looking images based totally on their content as opposed to image annotations. Study greater in: characteristic Extraction in content material-primarily based picture Retrieval. Retrieval of images based totally no longer on keywords or annotations but primarily based on features extracted directly from the image data. Study greater in: visible sample based compressed area image Retrieval. A method framework for correctly retrieving images from a set by means of similarity. The retrieval is predicated on extracting the ideal characteristic portions describing the desired contents of snap shots. in addition, appropriate querying, matching, indexing and looking techniques are required. Analyze more in: photograph classification and Retrieval with Mining technology. A technique framework for efficiently retrieving pix from a collection by similarity. The retrieval relies on extracting the ideal function quantities describing the desired contents of snap shots. Further, suitable querying, matching, indexing, and looking techniques are required. Research greater in: superior strategies for item-primarily based photo Retrieval. Picture indexing and retrieval strategies which use image contents, this is, low-stage (primitive) capabilities of an photo, consisting of color, shapes, textures, and so on. Queries also are furnished in a form of pix (sketches or picture examples). Research extra in: photo Retrieval practice and studies. This method retrieves or searches virtual images from big databases using the content of the snap shots themselves or syntactical photograph functions without human intervention. To useful resource photograph retrieval, techniques from facts, pattern recognition, signal processing, and computer imaginative and prescient are typically deployed. different phrases used interchangeably for CBIR are query by way of photograph content material (QBIC) and content material-primarily based visible records retrieval (CBVIR). Examine extra in: Linguistic Indexing of pictures with Database. Mediation. A system framework for efficiently retrieving photographs from a group by way of similarity. The retrieval is predicated on extracting the right function quantities describing the favored contents of images. similarly, suitable querying, matching, indexing and looking techniques are required. examine greater in: teaching and getting to know image publications with visual bureaucracy additionally called query by means of photo content material (QBIC), gives the technology permitting to organize digital pics with the aid of their visual functions. they are based totally at the software of pc vision techniques to the photo retrieval problem in big databases. contentbased picture Retrieval (CBIR) consists of retrieving the most visually similar photos to a given question image from a database of pix. analyze more in: the usage of global form Descriptors for content scientific-primarily based photograph Retrieval.

\section{Feature Extraction}

Feature extraction are very important step in image retrieving system in feature extraction method describing lots of descriptors. Color, texture, shape and layout are the property of many images in color image extraction searching the image with the color such as contrast. In shape property using the size and shapes such as correlation rectangle, circle symbols etc. In texture feature extraction using different parameters like entropy ,local range and standard deviation. photographs retrieval can be achieved from the virtual picture database on the premise of shade, form or texture. amongst a lot of these three capabilities aggregate of texture and coloration characteristic works very successfully in most conditions. in step with discern 1 while a query photo is submitted for photograph retrieval, its coloration and texture features are extracted and matching operation is finished between query photo functions and the picture capabilities saved in database.

$\mathrm{M}$

$\mathrm{ENT}=\sum \mathrm{P}_{\mathrm{z}} \log$

$\left(1 / \mathrm{P}_{\mathrm{k}}\right) \mathrm{k}=1$

where, $\mathrm{ENT}=$ Entropy of $\mathrm{I} / \mathrm{P} ; \mathrm{M}=$ Sample side; $\mathrm{P}=$ Probability

Table 1: Features Extraction

\begin{tabular}{|l|l|}
\hline Parameters & Formulas \\
\hline Entropy & $\mathrm{K}=1$ to $\mathrm{m}, \sum \mathrm{p}_{\mathrm{k}} \log 1 / \mathrm{p}_{\mathrm{k}}$ \\
\hline $\begin{array}{l}\text { Standard } \\
\text { deviation }\end{array}$ & $S=\sqrt{\frac{1}{N-1} \sum_{i=1}^{N}\left|A_{i}-\mu\right|^{2}}$, \\
\hline Local range & $\begin{array}{l}\text { imread('liftingbody.png'); } \\
\mathrm{J}=\operatorname{rangefilt}(\mathrm{I}) ;\end{array}$ \\
\hline contrast & $\mathrm{F} 1=\sum_{i, j=0}^{N-1} P_{i}, j(i-j) 2$ \\
\hline correlation & $\begin{array}{l}\mathrm{A}=[\mathrm{x} \text { y } 2 * \mathrm{y}+3] ; \\
\mathrm{R}=\text { corrcoef(A) }\end{array}$ \\
\hline
\end{tabular}

\subsection{Image Histogram}

An photograph histogram is a type of histogram that acts as a graphical illustration of the tonal distribution in a virtual picture. It plots the range of pixels for every tonal value. 


\section{International Journal of Science and Research (IJSR) \\ ISSN (Online): 2319-7064}

Index Copernicus Value (2013): 6.14 | Impact Factor (2015): 6.391

\subsection{Texture Feature Extraction}

Feature extraction is very crucial step in image retrieval system to describe the image with minimum number of descriptors [16]. Texture is an important property of many types of images. To extract the texture features, entropy, local range and standard deviation measures are used as performance parameters.

Texture $=($ Entropy + Standard deviation + local Range $)$

\subsection{Entropy}

\subsection{Standard Deviation}

The standard deviation value calculated as:

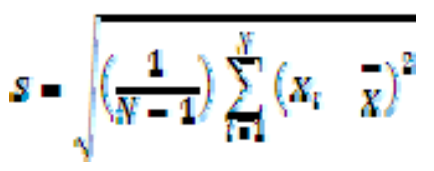

where $\mathrm{x}$ is the mean

\subsection{Local Range}

$\mathrm{LC}=$ (maximum value of chosen pixel-minimum value of chosen pixel).

\section{Content Based Image Retrieval (Proposed Work)}

Combined value of color, texture feature and searching work very effectively in most situations. This paper uses histogram, entropy, standard deviation and local range, contrast and correlation.

Image Retrieval $=$ Color feature + Texture Feature + layout + shape

According to Figure 1 when a query images is passing for image retrieval, its color feature are extracted with different parameters such as entropy, standard deviation, contrast, local range and correlation. Firstly load large amount of images in database and set the range(negative for add noise and 0 for $100 \%$ match)after that resize the image $[128,128]$ to get similar size of images, then convert RGB to GRAY and generate Histogram for color image, then calculated the parametric values and combine their image features into database. When a query image is loaded we apply the same procedure 2-11 to find the combine features of query image and then find the distance between the query image and database image, and then give the resulted image according to set range in GUI.

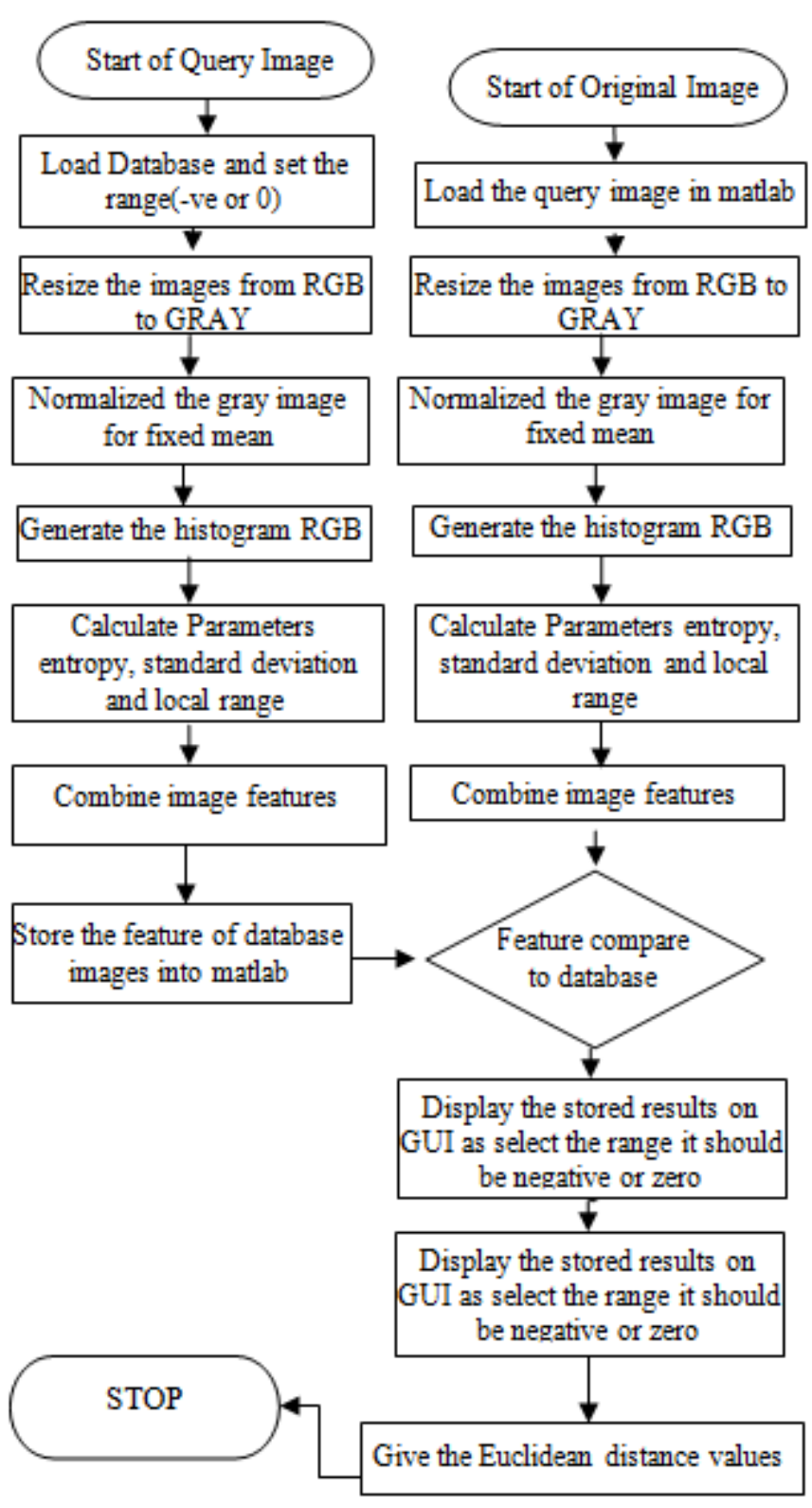

Figure 1: Flow chart for image retrieval system with different parameter values.

\section{Algorithms for Proposed Work}

Step 1: To close all pre-open windows.

Step 2: Load database in the Mat lab workspace.

Step 3: Resize the image for [128. 128].

Step 4: To initialize parametric values .

Step 5: To select range (-ve for add noise and 0 for $100 \%$ match).

Step 6: Convert image from RGB to Gray.

Step 7: Normalize the gray image for fixed mean.

Step 8: Generate the histogram of RGB.

Step 9: Find entropy, standard deviation and local range of Gray.

Step 10: Combine the image feature.

Step 11: Load the test image.

Step 12: Apply the procedure 2-11 to find combine feature of test image.

Step 13: Determine the normalized Euclidean distance of test image with stored image of database. 


\section{International Journal of Science and Research (IJSR) \\ ISSN (Online): 2319-7064}

Index Copernicus Value (2013): 6.14 | Impact Factor (2015): 6.391

Step14: Sort the normalized Euclidean distance values

Step 15: Display the result on GUI.

\section{Simulation Results}

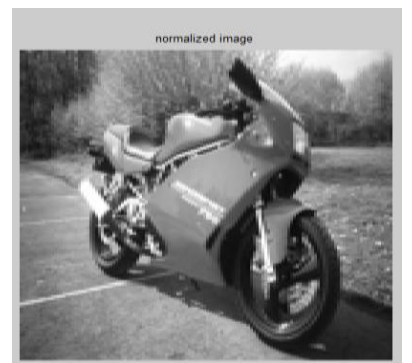

Figure 2: Normalized image

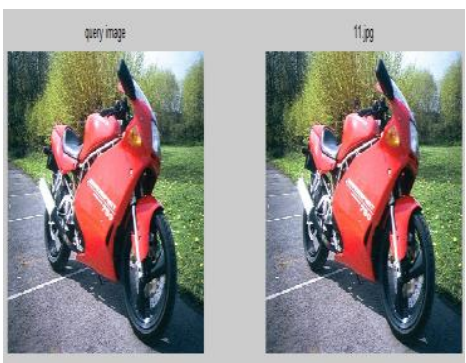

Figure 3: Resulted image

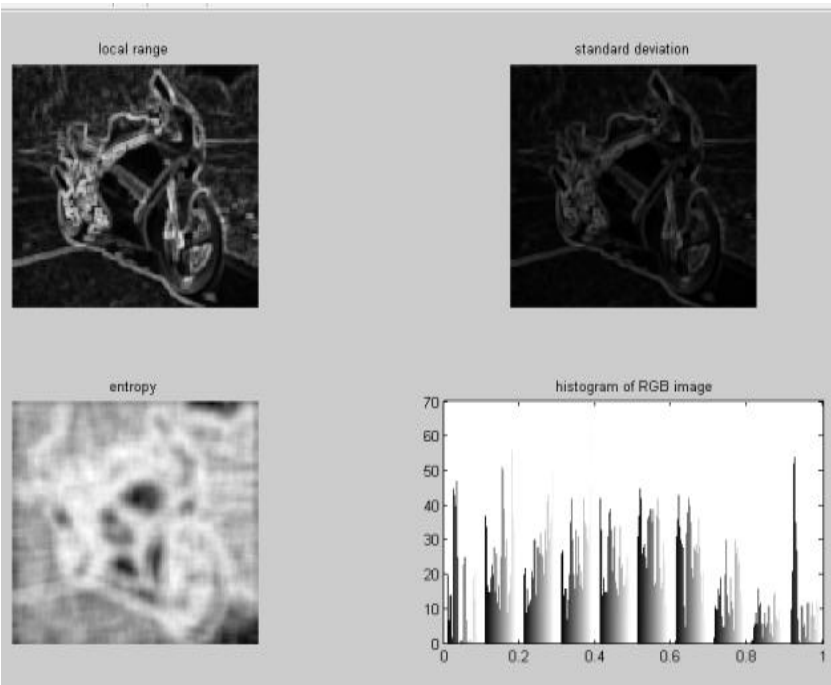

Figure 4: (Local range, Entropy, Standard deviation and Histogram)

Table 2: Simulation Result

\begin{tabular}{|c|c|c|c|c|}
\hline $\begin{array}{c}\text { Query } \\
\text { image }\end{array}$ & $\begin{array}{c}\text { Elapsed } \\
\text { time }\end{array}$ & Contrast & Correlation & Energy \\
\hline 1 & $\begin{array}{c}24.494920 \\
\mathrm{Sec}\end{array}$ & $3.1221 \mathrm{e}+003$ & 0.1750 & $7.4623 \mathrm{e}-005$ \\
\hline
\end{tabular}

\section{Conclusion and Future Work}

In this paper using query images that is search through the large database and calculate the elapsed time of searching the query image with the help of parameters, but for future work using a videos clips to searching the images through the large amount of database or enhance the searching elapsed time of searching images through database.

\section{References}

[1] N Gnaneswara Rao, Dr. Vijaya Kumar and V Venkata Krishna, "Texture Based Image Indexing and Retrieval", Published in IJCSNS International Journal of Computer Science and Network Security, VOL.9 No.5, May 20

[2] Raimondo Schettini, Gianluigi and Silvia, "A Survey of methods for color image indexing and retrieval in image database", Published in Istituto Tecnologie Infomatioche Multimediali Consilio Nazionale delle Ricerche Via Ampere 56, 20131 Milano Italy.

[3] Hui Yu, Mingjing Li and Hong-Jiang Zhang, "Color texture moments for content-based image retrieval" IEEE conference paper, 2002.

[4] Wasim Khan, Nilofar Khan and Shiv Kumar, "A Proposed Method for Image Retrieval using Histogram values and Texture Descriptor Analysis", Published in International Journal of Soft Computing and Engineering (IJSCE) ISSN: 2231-2307, Volume-I, Issue-II, May 2011.

[5] A W M Smeulders, M Worring, S Santini and A Gupta, "Content Based image Retrieval at the End of the Early Years" Published in IEEE Transactions on Pattern Analysis and Machine Intelligence Volume: 22 , Issue: 12, Dec 2000.

[6] Hossein Nezamabadi-pour and Saeid Saryazdi, "ObjectBased Image Indexing and Retrieval in DCT Domain using Clustering Techniques" Published in International Journal of Computer, Electrical, Automation, Control and Information Engineering Vol:1, No:3, 2007.

[7] Greg Pass and Ramin Zabih, "Histogram Refinement for Content-Based Image Retrieval" Published in Computer Science Department Cornell University Ithaca, NY 14853.

[8] Dr. Divakar Yadav and Divya Ragatha Venkata, "Image Query Based Search Engine using Image Content Retrieval", Published in 2012 14th International Conference on Modelling and Simulation.

[9] Mohammad Atique and Amol P Bhagat, "Design and Development of Systems for Image Segmentation and Content Based Image Retrieval", Published in Computational Intelligence and Signal Processing (CISP), 2nd National Conference on 2-3 March 2012.

[10]Ryszard S Choras, "Image Feature Extraction Techniques and Their Applications for CBIR and Biometrics Systems", Published in International Journal Of Biology And Biomedical Engineering, Issue

[11] X. Zhou and T. S. Huang, "Relevance feedback in image retrieval: a comprehensive review," ACM Multimedia Systems Journal, special issue on CBIR, 8(6): pp. 536-544, 2003.Vol. 1, 2007.

[12] J. Yu and Q. Tian, "Adaptive discriminate projection for content-based image retrieval", Proc. of Intl. Conf. on Pattern Recognition, Hong Kong, August 2006.

\section{Author Profile}

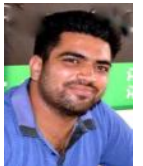

Vinay Thakur is currently with Sri Sai University Palampur, Himachal, INDIA working as Asst Professor in Department of ECE. He has 5 year experience in teaching .He did his B.Tech and M.Tech

Volume 5 Issue 6, June 2016 www.ijsr.net 


\section{International Journal of Science and Research (IJSR) \\ ISSN (Online): 2319-7064}

Index Copernicus Value (2013): 6.14 | Impact Factor (2015): 6.391

from Lovely Professional University Jalandhar. His area of interest is image processing, information retrieval and signal.

Ritika Verma has done B.Tech (I.T) from BUEST in 2014 and M.Tech (CSE) from Sri Sai University Palampur in 2016. Her areas of interest are image retrieval system, image processing and data mining.

Volume 5 Issue 6, June 2016 www.ijsr.net

Licensed Under Creative Commons Attribution CC BY 